\title{
Simple Approximations of Semialgebraic Sets and their Applications to Control
}

\author{
Fabrizio Dabbene $^{\mathrm{a}}$, Didier Henrion ${ }^{\mathrm{b}, \mathrm{c}}$ and Constantino Lagoa ${ }^{\mathrm{d}}$ \\ ${ }^{a}$ CNR-IEIIT; c/o Politecnico di Torino; C.so Duca degli Abruzzi 24, Torino; Italy \\ ${ }^{\mathrm{b}} L A A S-C N R S$, Université de Toulouse, CNRS, Toulouse, France. \\ ${ }^{\mathrm{c}}$ Faculty of Electrical Engineering, Czech Technical University in Prague, Technická 2, CZ-16626 Prague, Czech Republic. \\ ${ }^{\mathrm{d}}$ Electrical Engineering Department, The Pennsylvania State University, University Park, PA 16802, USA.
}

\begin{abstract}
Many uncertainty sets encountered in control systems analysis and design can be expressed in terms of semialgebraic sets, that is as the intersection of sets described by means of polynomial inequalities. Important examples are for instance the Schur and Hurwitz stability domains. These sets often have very complicated shapes (nonconvex, and even non-connected), which renders difficult their manipulation. It is therefore of considerable importance to find simple-enough approximations of these sets, able to capture their main characteristics while maintaining a low level of complexity. For these reasons, in the past years several convex approximations, based for instance on hyperrectangles, polytopes, or ellipsoids have been proposed.

In this work, we move a step further, and propose possibly nonconvex yet still simple approximations, based on a small volume polynomial superlevel set of a single positive polynomial of given degree. We show how these sets can be easily approximated by minimizing the $L^{1}$ norm of the polynomial over the semialgebraic set, subject to positivity constraints. Intuitively, this corresponds to the trace minimization heuristic commonly encountered in minimum volume ellipsoid problems. From a computational viewpoint, we design a hierarchy of convex linear matrix inequality problems to generate these approximations, and we provide theoretically rigorous convergence results, in the sense that the hierarchy of outer approximations converges in volume (or, equivalently, almost everywhere and almost uniformly) to the original set.

Finally, we show how the concept of polynomial superlevel set can be used to generate samples uniformly distributed on a given semialgebraic set. The efficiency of the proposed approach is demonstrated by different numerical examples.
\end{abstract}

Key words: Semialgebraic set, Approximation, Sampling

\section{Introduction}

In this paper, we address the problem of how to determine "simple" approximations of semialgebraic sets in Euclidean space, and we show how these approximations can be exploited to address several problems of interest in systems and control. To be more precise, given a set

$$
\mathcal{K} \doteq\left\{x \in \mathbb{R}^{n}: g_{i}(x) \geq 0, i=1,2, \ldots, m\right\}
$$

which is compact, with non-empty interior and described by real multivariate polynomials $g_{i}(x), i=1,2, \ldots, m$,

\footnotetext{
* This work was partially supported by the National Science Foundation under grants CNS-1329422 and ECCS-1201973.

Email addresses: fabrizio.dabbene@ieiit.cnr.it (Fabrizio Dabbene), henrion@laas.fr (, Didier Henrion), lagoa@engr.psu.edu (and Constantino Lagoa).
}

and given a compact set $\mathcal{B} \supset \mathcal{K}$, we aim at determining a so-called polynomial superlevel set (PSS)

$$
\mathcal{U}(p) \doteq\{x \in \mathcal{B}: p(x) \geq 1\}
$$

that constitutes a good outer approximation of the set $\mathcal{K}$ of interest and converges in a mathematically welldefined way to $\mathcal{K}$ when increasing the degree of the real multivariate polynomial $p$ to be found.

In particular, the proposed PSS is based on an easily computable polynomial approximation of the indicator function of the set $\mathcal{K}$. In the paper, we show that suitable approximations of the indicator function can be obtained by solving a convex optimization problem whose constraints are linear matrix inequalities (LMIs) and that, as the degree of the approximation increases, these converge in $L^{1}$-norm, almost uniformly and almost ev- 
erywhere to the indicator function of the semialgebraic set $\mathcal{K}$ of interest. Moreover, the set approximations provided in this paper can be thought as a direct generalization of classical ellipsoidal set approximations, in the sense that if second degree approximations are used, we exactly recover well-known approaches.

The main motivation for the problem addressed in the paper is the fact that semialgebraic sets are frequently encountered in control. As an example, consider the Hurwitz or Schur stability regions of a polynomial. It is a well-known fact that the these regions are semialgebraic sets in the coefficient space. The polynomial inequalities that define these stability sets can be derived from wellknown algebraic stability criteria. Another motivating example is related to set-based filtering. In the paper [11], the approach discussed here is used to derive randomized approximations of the image set of nonlinear discrete-time systems, with applications to nonlinear filtering. Also, robust $\mathcal{H}_{\infty}$ control under nonlinear (polynomial uncertainty) can be formulated in this framework. The approach taken in this paper is the following: given the set $\mathcal{K}$, we search for a minimum volume PSS that contains the set $\mathcal{K}$. Since there is in general no analytic formula for the volume of a semialgebraic set, in terms of the coefficients of the polynomials defining the set, ${ }^{1}$ it is very challenging to solve this optimization problem locally, let alone globally. Instead, the main contribution of this paper is to describe and justify analytically and geometrically a computationally tractable heuristic based on $L^{1}$-norm minimization. Second, we show that the same approach can be employed to obtain the largest (in terms of the $L^{1}$ surrogate for the volume) PSS inscribed in $\mathcal{K}$. Moreover, it is shown how the ideas put forth in this paper can be used to address the important problem of generating samples uniformly distributed over a semialgebraic set. Examples of applications in a systems analysis and controller design context are also provided.

The work presented in this paper is an extension of the preliminary results discussed in the conference papers [9] and [10], and it provides a more in depth analysis of both theoretical and implementation aspects.

\subsection{Previous work and related literature}

The necessity of approximating overly complicated sets by means of simpler and easy manageable geometrical shapes arises in different research fields such as optimization, system identification and control. In particular, in the systems and control community, the most common approach is to introduce outer bounding sets, that is sets of minimum size which are guaranteed to

\footnotetext{
1 See however reference [26] which explains how explicit formulas can be obtained with discriminants in exceptional cases.
}

contain the set to be approximated. For instance, in the context of robust filtering, set-theoretic state estimators for uncertain nonlinear dynamic systems have been proposed in $[1,15,17,31]$. These strategies adopt a setmembership approach [16,30], and construct (the smallest) compact set guaranteed to bound the system states that are consistent with the measured output and the norm-bounded uncertainty. The most common geometrical shape adopted in these work is the ellipsoidal one, for the double reason that it has a very simple description - the center and the shape matrix are sufficient to provide a complete characterization - and that its determination usually can be formulated as a convex (usually quadratic) optimization problem. The use of ellipsoidal sets in the state estimation problems was introduced in the pioneering work [30] and used by many different authors from then on; see, for example, [15,17]. Similarly, inner approximations are employed for instance in the solution of design centering problems [34] and for fixedorder controller design [21]. In this case, one aims at constructing the set largest size inscribed in the set of interest.

Besides ellipsoids, other shapes have been considered in the recent literature. The use of polyhedra was proposed in [22] to obtain an increased estimation accuracy, while zonotopes have been also recently studied in $[1,18]$. In $[6]$ a heuristic based on polynomial optimization and convex relaxations is proposed for computing small volume polytopic outer approximations of a compact semialgebraic set. More recent works, like for instance [21,25], employ sets defined by semialgebraic conditions.

All these works, however, make use of approximating sets which are convex: to the best of our knowledge the present paper represents the first rigorous and general approach to construct approximations which are nonconvex, yet still simple enough.

\section{Problem statement}

\subsection{Notation}

The notation $A \succ 0(\succeq 0)$ means that the symmetric matrix $A$ is positive definite (semidefinite), and given two matrices $A$ and $B$ we write $A \succeq B$ whenever $A-B \succeq 0$. Given a set $\mathcal{K} \subset \mathbb{R}^{n}$, its indicator function is defined as $\mathbb{I}_{\mathcal{K}}(x) \doteq\left\{\begin{array}{ll}1 & \text { if } x \in \mathcal{K} \\ 0 & \text { if } x \notin \mathcal{K}\end{array}\right.$ and its volume or, more precisely, the Lebesgue measure of $\mathcal{K}$, is denoted by vol $\mathcal{K} \doteq$ $\int_{\mathcal{K}} d x=\int_{\mathbb{R}^{n}} \mathbb{I}_{\mathcal{K}}(x) d x$. The set of all real coefficient polynomials of degree less than or equal to $d$ is denoted by $P_{d}$. The monomial basis for this set is represented by the (column) vector $\pi_{d} \in P_{d}$, so that any $p \in P_{d}$ can be expressed as: $p(x)=\pi_{d}^{T}(x) p=\pi_{\lceil d / 2\rceil}^{T}(x) P \pi_{\lceil d / 2\rceil}(x)$,where 
$p$ is a real (column) vector ${ }^{2}$ and $P$ is a symmetric matrix of appropriate size, often referred to as Gram matrix. Finally, given a compact set $\mathcal{B}$ and a polynomial $p \in P_{d}$ positive on $\mathcal{B}$, define its $L^{1}$ norm over $\mathcal{B}$, denoted by $L_{\mathcal{B}}^{1}$ or just $L^{1}$ when the set $\mathcal{B}$ used is clear from the context, as $\|p\|_{1} \doteq \int_{\mathcal{B}} p(x) d x$.

\subsection{Problem Statement}

With the notation defined above, we are now ready to define the central problem in this paper. We consider the basic semialgebraic set $\mathcal{K}$ defined in (1), which is assumed to be compact and with a non-empty interior.

As discussed in the Introduction, the set $\mathcal{K}$ has typically a complex description in terms of its defining polynomials (e.g. coming from physical measurements and/or estimations). For this reason, we aim at finding a "simpler" approximation of this set which has enough degrees of freedom to capture its characteristics. This approximation is the polynomial superlevel set (PSS) $\mathcal{U}(p)$ defined in (2) in terms of a real multivariate polynomial $p \in P_{d}$ of given degree $d$. This degree controls the complexity of the approximation. Among the family of possible PSS that can be constructed, we search for the one that provides the set $\mathcal{U}(p)$ of minimum volume, while containing the set of interest $\mathcal{K}$, hence capturing most of its geometric features. Formally, we define the following optimization problem

Problem 1 (Minimum volume outer PSS) Given $d \in \mathbb{N}$ and a compact semialgebraic set $\mathcal{K}$, find a polynomial $p \in P_{d}$ whose $\operatorname{PSS\mathcal {U}}(p)$ is of minimum volume and contains $\mathcal{K}$. That is, solve the following optimization problem

$$
v_{d}^{*} \doteq \inf _{p \in P_{d}} \operatorname{vol} \mathcal{U}(p) \quad \text { s.t. } \quad \mathcal{K} \subseteq \mathcal{U}(p)
$$

Note that this problem can be viewed as the natural extension of the problem of computing the minimum volume ellipsoid containing $\mathcal{K}$. Indeed, if $\mathcal{K}$ is convex and the polynomial $p$ is quadratic $(d=2)$, then the infimum of problem (3) is attained, and the optimal set $\mathcal{U}(p)$ is given by the unique (convex) ellipsoid of minimum volume that contains $\mathcal{K}$, called Löwner-John ellipsoid. In particular, if $\mathcal{K}$ is the convex-hull of a finite set of points, this ellipsoid can be computed by convex optimization, see e.g. $[4, \S 4.9]$.

We remark however that, for $d$ greater than 2 , the optimization problem (3) is nonlinear and semi-infinite, in the sense that the optimization is over the finitedimensional vector space $P_{d}$, but subject to an infinite

\footnotetext{
2 Note that we use $p$ to denote both the polynomial and the vector of its coefficients whenever no ambiguity is possible.
}

number of constraints, necessary to cope with the set inclusion.

Theorem 1 The sequence of infima of problem (3) monotically converges from above to vol $\mathcal{K}$, i.e. for all $d \geq 1$ it holds $v_{d}^{*} \geq v_{d+1}^{*}$ and $\lim _{d \rightarrow \infty} v_{d}^{*}=\operatorname{vol} \mathcal{K}$.

The proof of the theorem is given in Appendix A.

\section{$3 \quad L^{1}$-norm minimization}

It is assumed that a "simple set" $\mathcal{B} \subset \mathbb{R}^{n}$ containing $\mathcal{K}$ is known. Here, by "simple" we mean that analytic expressions of the moments of the Lebesgue measure on $\mathcal{B}$ should be available, so that integration of polynomials can be carried out readily. In the following, we assume that the set $\mathcal{B}$ is an $n$-dimensional hyperrectangle of the form

$$
\mathcal{B}=[a, b] \doteq\left\{x \in \mathbb{R}^{n}: a_{i} \leq x_{i} \leq b_{i}, i=1, \ldots, n\right\}
$$

with $a$ and $b$ given vectors of $\mathbb{R}^{n}$. This is a very mild assumption since, given a semialgebraic set like the set $\mathcal{K}$ above, one can easily compute an hyperrectangle containing it; see e.g. [6]. We note that more complex sets $\mathcal{B} \supseteq \mathcal{K}$ can be considered, provided that integration of polynomials over them is easily done; see Remark 3.

Assume now, without loss of generality, that the polynomial $p$ used to build the PSS is non-negative on $\mathcal{B}$. Then, observe that by definition of PSS (see Figure 1 for an illustration) we have $p \geq \mathbb{I}_{\mathcal{U}(p)}$ on $\mathcal{B}$. Hence, integrating

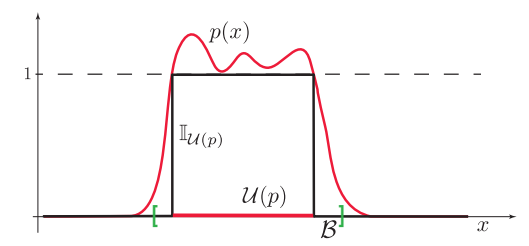

Fig. 1. Illustration of Chebychev's inequality: the polynomial is always greater or equal than the indicator function of $p(x) \geq 1$, hence the integral of $p$ over $\mathcal{B}$ is always an upper bound of the volume of $\mathcal{U}(p)$.

both sides we get the following inequality

$$
\int_{\mathcal{B}} p(x) d x \geq \int_{\mathcal{B}} \mathbb{I}_{\mathcal{U}(p)}(x) d x=\operatorname{vol} \mathcal{U}(p) .
$$

This inequality is indeed widely used in probability, where it goes under the name of Chebyshev's inequality, see e.g. $[2, \S 2.4 .9]$. Note that, since the polynomial $p$ is nonnegative on $\mathcal{B}$, then the left-hand side of inequality (5) corresponds to the $L^{1}$-norm of $p$ on $\mathcal{B}$, so that the inequality simply becomes

$$
\|p\|_{1} \geq \operatorname{vol} \mathcal{U}(p) .
$$


These derivations motivate us to the formulation of the following $L^{1}$-norm minimization problem, which we choose as a surrogate of the original minimum volume outer PSS introduced in Problem 1.

Problem 2 (Minimum $L^{1}$-norm outer PSS) Given a semialgebraic set $\mathcal{K}$, a bounding set $\mathcal{B} \supseteq \mathcal{K}$, and a degree $d$, solve the optimization problem

$$
\begin{aligned}
w_{d}^{*} \doteq & \inf _{p \in P_{d}}\|p\|_{1} \\
& \text { s.t. } \quad p \geq 0 \text { on } \mathcal{B}, p \geq 1 \text { on } \mathcal{K} .
\end{aligned}
$$

Note that a $L^{1}$-norm minimization approach was originally proposed in [20] for the numerical computation of the volume and of the higher order moments of a semialgebraic set. The intuition underlying the formulation of Problem 2 is similar. We now elaborate on some of the characteristics of the minimum $L^{1}$-norm outer PSS problem defined above. First note that, for fixed $d$, when solving Problem 2 we are minimizing an upper-bound on the volume of the PSS. Thus, the solution is expected to be a good approximation of the set $\mathcal{K}$. Second, it can be shown that, as the degree $d$ increases, the Chebyshev bound (6) becomes increasingly tight. Indeed, the following fundamental result shows that the proposed solution converges to the minimum volume outer PSS.

Theorem 2 Given $d \in \mathbb{N}$, the infimum in problem (7) is attained for a polynomial $p_{d}^{*} \in P_{d}$. Moreover, $w_{d}^{*} \geq v_{d}^{*}$ and $\mathcal{U}\left(p_{d}^{*}\right) \supseteq \mathcal{K}$. Finally $w_{d}^{*} \geq w_{d+1}^{*}$ and $\lim _{d \rightarrow \infty} w_{d}^{*}=$ $\lim _{d \rightarrow \infty} v_{d}^{*}=\operatorname{vol} \mathcal{K}$.

The proof of Theorem 2, which represents one of the main contributions of the paper, is given in Appendix B. Some remarks are at hand regarding this result.

\section{Remark 1 (Convergence almost everywhere)}

Theorem 2 implies that, for high enough order of approximation, the PSS obtained by minimizing the $L^{1}$-norm of the polynomial defining it can be "arbitrarily close" to the semialgebraic set of interest. More precisely, as $d \rightarrow \infty$, $\left\|p_{d}^{*}\right\|_{1}$ and, as a consequence $\operatorname{vol} \mathcal{U}\left(p_{d}^{*}\right)$, converges to $\operatorname{vol} \mathcal{K}$. Since $\mathcal{K} \subseteq \mathcal{U}\left(p_{d}^{*}\right)$, it follows that the Lebesgue measure of the difference between these sets converges to zero. In other words, one has almost everywhere convergence. From Theorems 2.5.1 and 2.5.3 in [2] the convergence is also almost uniform, up to extracting a subsequence.

Remark 2 (Relationship with ellipsoidal trace minimization) Since the $L^{1}$ norm represents an upper bound of the volume of the PSS, its minimization leads to a minimization of the volume. Moreover, the use of the $L^{1}$-norm PSS has a nice geometric interpretation, which further justifies its use. To this end, we first note that the objective function in (7) reads

$$
\begin{aligned}
\|p\|_{1} & =\int_{\mathcal{B}} p(x) d x=\int_{\mathcal{B}} \pi_{\delta}^{T}(x) P \pi_{\delta}(x) d x \\
& =\operatorname{trace}\left(P \int_{\mathcal{B}} \pi_{\delta}(x) \pi_{\delta}^{T}(x) d x\right)=\operatorname{trace} P M
\end{aligned}
$$

where

$$
M \doteq \int_{\mathcal{B}} \pi_{\delta}(x) \pi_{\delta}^{T}(x) d x
$$

is the matrix of moments of the Lebesgue measure on $\mathcal{B}$ in the basis $\pi_{\delta}(x)$. Note that, if the basis in equation (8) is chosen such that its entries are orthonormal with respect to the (scalar product induced by the) Lebesgue measure on $\mathcal{B}$, then $M$ is the identity matrix and inequality (6) becomes

$$
\text { trace } P \geq \operatorname{vol} \mathcal{U}(p)
$$

which indicates that, under the above constraints, minimizing the trace of the Gram matrix $P$ entails minimizing the volume of $\mathcal{U}(p)$. It is important to remark that, in the case of quadratic polynomials, i.e. $d=2$, we retrieve the classical trace heuristic used for volume minimization of ellipsoids, see e.g. [14]. Indeed, if $\mathcal{B}=[-1,1]^{n}$, then the basis $\pi_{1}(x)=\frac{\sqrt{6}}{2} x$ is orthonormal with respect to the Lebesgue measure on $\mathcal{B}$ and $\|p\|_{1}=\frac{3}{2}$ trace $P$. Moreover, note that the constraint that $p$ is nonnegative on $\mathcal{B}$ implies that the curvature of the boundary of $\mathcal{U}(p)$ is nonnegative, hence that $\mathcal{U}(p)$ is convex. Thus, $\mathcal{U}(p)$ is indeed an ellipsoid.

\section{LMI hierarchy to compute the PSS}

In this section, we provide the basic details on the numerical computation of the solution of the minimum $L^{1}$ norm PSS introduced in Problem 2. The proposed approach consists of using sums of squares (SOS) representations of the polynomial positivity constraints in Problem 2 . Denote by $\Sigma_{2 d}$ the set of polynomials $p \in P_{2 d}$ that can be represented as sums of squares of other polynomials, i.e.

$$
p=\sum_{k=1}^{n_{p}} p_{k}^{2}, \quad p_{k} \in P_{d}, k=1, \ldots, n_{p}
$$

In problem (7), we aim at finding a polynomial $p \in P_{d}$ such that i) $p$ is positive on $\mathcal{B}$, and ii) $p-1$ is positive on $\mathcal{K}$. In order to obtain a numerically solvable problem, we enforce positivity by requiring the polynomial to be SOS, and use Putinar's Positivstellensatz; e.g., see $[28,23,7,27]$. More precisely, fix $r \in \mathbb{N}$, and consider the 
problem

$$
\begin{aligned}
w_{2 r, d}^{*}= & \min _{p \in P_{d}} \int_{\mathcal{B}} p(x) d x \\
& \text { s.t. } \\
& p(x)=s_{0, \mathcal{B}}(x)+\sum_{j=1}^{n} s_{j, \mathcal{B}}(x)\left(x_{j}-a_{j}\right)\left(b_{j}-x_{j}\right) \\
& s_{0, \mathcal{B}} \in \Sigma_{2 r} \\
& s_{j, \mathcal{B}} \in \Sigma_{2(r-1)}, \quad j=1,2, \ldots, n \\
& p(x)-1=s_{0, \mathcal{K}}(x)+\sum_{i=1}^{m} s_{i, \mathcal{K}}(x) g_{i}(x) \\
& s_{0, \mathcal{K}} \in \Sigma_{2 r} \\
& s_{i, \mathcal{K}} \in \Sigma_{2\left(r-r_{i}\right)}, \quad i=1,2, \ldots, m .
\end{aligned}
$$

where $r_{i}$ is the smallest integer greater than half the degree of $g_{i}$ for $i=1,2, \ldots, m$. It should be noted that the objective function of problem (9) is an easily computable linear function of the coefficients of the polynomial $p$. Moreover, this problem is a particular instance of a semidefinite problem and the constraints can be recast in terms of linear matrix inequalities; see, for instance, [23]). Several MatLab toolboxes have efficient and easy to use interfaces to model problems of the form above; e.g., see YALMIP [24]. The problem formulated above is an LMI with a number of variables approximately equal to $n_{v a r}=(m+n)\left(\begin{array}{c}n+2 r \\ 2 r\end{array}\right)$. This problem can be solved at given accuracy in a time which is a polynomial function of $n_{v a r}$. See e.g. [4, Section 6.6.3] for a detailed analysis of the complexity of solving LMI problems.

Not only we can numerically solve problem (9), but the following theorem, which is an immediate consequence of the results in [28], holds.

Theorem 3 Let us denote by $p_{2 r, d}^{*}$ a solution of problem (9). Then, the following hold

i) for each $d \in \mathbb{N}$, the value of problem (9) converges to the value of problem (7) as $r \rightarrow \infty$, i.e. $\lim _{r \rightarrow \infty} w_{2 r, d}^{*}=w_{d}^{*}$,

ii) for any $2 r \geq d, p_{2 r, d}^{*} \geq 0$ on $\mathcal{B}$,

iii) for any $2 r \geq d, p_{2 r, d}^{*} \geq 1$ on $\mathcal{K}$.

We conclude that $p_{2 r, d}^{*}$ can be used to compute a PSS approximation for $\mathcal{K}$. For our numerical examples, we have used the YALMIP [24] interface for Matlab to model the LMI optimization problem (9) and the SDP solver SeDuMi [32] to numerically solve the problem. Since the degrees of the semialgebraic sets we compute are typically low (say less than 20), we did not attempt to use alternative polynomial bases (e.g. Chebyshev polynomials) to improve the quality and resolution of the optimization problems; see [20] for a discussion on these nu- merical matters in the context of semialgebraic set volume approximation.

\section{Remark 3 (Computation of Bounding Box $\mathcal{B}$ )}

We remark that an outer-bounding hyper-rectangle $\mathcal{B}=[a, b]$ of a given semialgebraic set $\mathcal{K}$ can be computed by solving relaxations of the following polynomial optimization problems

$$
\begin{aligned}
a_{j} & =\arg \min _{x \in \mathbb{R}^{n}} x_{j} \text { subject to } x \in \mathcal{K}, \quad j=1, \ldots, n, \\
b_{j} & =\arg \max _{x \in \mathbb{R}^{n}} x_{j} \text { subject to } x \in \mathcal{K}, \quad j=1, \ldots, n,
\end{aligned}
$$

which compute the minimum and maximum value of each component of the vector $x$ over the semialgebraic set $\mathcal{K}$. Moreover, the problem above can be recast as an $L M I$ optimization problem, as well explained in [6, Remark 1]. We also remark that convergence will depend on the specific choice of $\mathcal{B}$; however, its effect cannot reasonably be quantified.

Remark 4 (Inner approximations) The proposed approach can be extended to derive inner approximations of the set $\mathcal{K}$, in the spirit of [8,19,21]. The idea is to construct an optimal outer PSS of the complement set

$$
\begin{aligned}
\overline{\mathcal{K}} \doteq \mathcal{B} \backslash \mathcal{K}=\left\{x \in \mathbb{R}^{n}: g_{1}(x)<0 \text { or } \cdots \text { or } g_{m}(x) \leq 0\right. \\
i=1,2, \ldots, m\} \cap \mathcal{B} \\
=\left(\mathcal{K}_{1} \cup \mathcal{K}_{2} \cup \cdots \cup \mathcal{K}_{m}\right) \cap \mathcal{B}
\end{aligned}
$$

with $\mathcal{K}_{j} \doteq\left\{x \in \mathbb{R}^{n}: g_{j}(x)<0\right\}$. Note that, since the set whose indicator function we want to approximate is a union of basic semialgebraic sets, the $L^{1}$ optimization problem to be solved becomes

$$
\begin{array}{ll}
\min _{p \in P_{d}} & \|p\|_{1} \\
\text { s.t. } & p \geq 0 \text { on } \mathcal{B} \\
& \vdots \\
& p \geq 1 \text { on } \mathcal{K}_{m} .
\end{array}
$$

Let $p_{d}^{*}$ attain the minimum, then the corresponding optimal inner approximation is given by the polynomial sublevel set $\mathcal{V}\left(p_{d}^{*}\right) \doteq\left\{x \in \mathcal{B}: p_{d}^{*}(x) \leq 1\right\}$.In this case, one can think of the polynomial $1-p_{d}^{*}$ as a lower bound for the indicator function of the set $\mathcal{K}$.

\section{$5 \quad$ Numerical examples}

In this section, we present several examples that illustrate the performance of the proposed approach.

\subsection{Discrete-time stabilizability region}

As a control-oriented illustration of the PSS approximation described in this paper, consider [19, Exam- 
ple 4.4] which is a degree 4 discrete-time polynomial $z \in \mathbb{C} \mapsto x_{2}+2 x_{1} z-\left(2 x_{1}+x_{2}\right) z^{3}+z^{4}$ to be stabilized by means of 2 real control parameters $x_{1}, x_{2}$. In other words, we are interested in approximating the set $\mathcal{K}$ of values of $x_{1}, x_{2}$ such that this polynomial has its roots with modulus less than one. An explicit basic semialgebraic description of the stabilizability region is built using the Schur stability criterion, resulting in the following basic semialgebraic set:

$$
\begin{aligned}
\mathcal{K}=\{x & \in \mathbb{R}^{2}: g_{1}(x)=1+2 x_{2} \geq 0, \\
g_{2}(x) & =2-4 x_{1}-3 x_{2} \geq 0, \\
g_{3}(x) & =10-28 x_{1}-5 x_{2}-24 x_{1} x_{2}-18 x_{2}^{2} \geq 0, \\
g_{4}(x) & \left.=1-x_{2}-8 x_{1}^{2}-2 x_{1} x_{2}-x_{2}^{2}-8 x_{1}^{2} x_{2}-6 x_{1} x_{2}^{2} \geq 0\right\} .
\end{aligned}
$$

This set is nonconvex and it is included in the box $\mathcal{B}=[-0.8,0.6] \times[-0.5,1.0]$. In Figure 2 we represent the PSS outer and inner approximations for different values of $d$. With the help of the YALMIP interface and the MOSEK semidefinite programming solver running on Matlab on a standard desktop PC, these polynomials could be computed in a matter of a few seconds.

\subsection{PID stabilizability region}

We now turn our attention to an example related to fixed order controller design. Consider [5, Example 2.2], in which the authors examine the problem of stabilizing the plant $P(s)=\frac{N(s)}{D(s)}$ where

$N(s)=s^{3}-2 s^{2}-s-1$
$D(s)=s^{6}+2 s^{5}+32 s^{4}+26 s^{3}+65 s^{2}-8 s+1$.

by means of a PID controller of the form $K_{\mathrm{PID}}(s)=$ $k_{\mathrm{P}}+\frac{k_{\mathrm{I}}}{s}+k_{D} s$. In particular, they are interested in finding the set of stabilizing PID gains, that is the set of gains for which the closed-loop characteristic polynomial $s D(s)+$ $\left(k_{\mathrm{I}}+k_{\mathrm{P}} s+k_{\mathrm{D}} s^{2}\right) N(s)$ is Hurwitz. For this special class of controllers, the authors provide a method based on the so-called signature of a set of properly constructed polynomials to determine the set of all PID gains that stabilize the plant. One should note that this procedure is not easily generalizable to more general classes of fixed order controllers.

In our setup, we are interested in approximating the set

$\mathcal{K}=\left\{x \in \mathbb{R}^{3}: s D(s)+\left(k_{\mathrm{I}}+k_{\mathrm{P}} s+k_{\mathrm{D}} s^{2}\right) N(s)\right.$ is Hurwitz, $\left.k_{\mathrm{I}}=25\left(x_{1}-1\right), k_{\mathrm{P}}=10\left(x_{2}-1.5\right), k_{\mathrm{D}}=10\left(x_{3}-1\right)\right\}$

with bounding box $\mathcal{B}=[-1,1]^{3}$. As one can see in Figure 4, the approached proposed in this paper provides a very good approximation of the set of stabilizing gains, even for a PSS of relatively low order $(d=$ 14). Again, employing the YALMIP interface and the MOSEK semidefinite programming solver running on Matlab, these polynomials were be computed in a matter of a few seconds on a standard desktop PC.

\section{Uniform sampling over semialgebraic sets}

In this section, we consider a problem that can be seen as the dual of the one considered in the previous section; that is, instead of trying to reconstruct/approximate the indicator function of an unknown set from points belonging to its interior, we aim at developing systematic procedures for generating uniformly distributed samples in a given set. This is an important problem, since many system specifications lead to sets described by a set of polynomial inequalities, and being able to draw samples from these type of sets provides the means for the design of systems with a complex set of specifications. In particular, the algorithm presented in this section can be used to generate uniform samples in the solution set of LMIs.

As before, we assume that the set of interest is a compact basic semialgebraic set defined as in (1), and that there exists a bounding hyper-rectangle $\mathcal{B}=[a, b]$ of the form (4). Then, the problem we discuss in this section is the following.

Problem 3 (Uniform Sample Generation over $\mathcal{K}$ ) Given a semialgebraic set $\mathcal{K}$ defined in (1) of nonzero volume, generate $N$ independent identically distributed (i.i.d.) random samples $x^{(1)}, \ldots, x^{(N)}$ uniformly distributed in $\mathcal{K}$.

Let us start by describing the approach proposed to solve this problem. First, we define the uniform density over the set $\mathcal{K}$ as $\mathbb{U}_{\mathcal{K}} \doteq \frac{\mathbb{I}_{\mathcal{K}}}{\text { vol } \mathcal{K}}$ where $\mathbb{I}_{\mathcal{K}}$ is the indicator function of the set $\mathcal{K}$. Then, the idea at the basis of the proposed method is to use a PSS approximation of the set $\mathcal{K}$ or, equivalently, a polynomial over approximation of the indicator function $\mathbb{I}_{\mathcal{K}}$, obtained employing the framework introduced in Sections 2 and 3.

To this end, given a degree $d \in \mathbb{N}$, consider the optimization problem (7) and let $p_{d}^{*}$ be a polynomial that achieves the optimum. If one examines the proof of Theorem 2 , one can see that this polynomial has the following properties

i) $p_{d}^{*} \geq \mathbb{I}_{\mathcal{K}}$ on $\mathcal{B}$

ii) As $d \rightarrow \infty, p_{d}^{*} \rightarrow \mathbb{I}_{\mathcal{K}}$ both in $L^{1}$ and almost uniformly on $\mathcal{B}$.

Hence, $p_{d}^{*}$ can arbitrarily approximate (from above) the indicator function of the set $\mathcal{K}$ and, therefore, it represents a so-called "dominating density" of the uniform density $\mathbb{U}_{\mathcal{K}}$ on $\mathcal{B}$. More formally, there exists a value $\beta>0$ such that $\beta p_{d}^{*}(x) \geq \mathbb{U}_{\mathcal{K}}(x)$ for all $x \in \mathcal{B}$. Hence, the rejection method from a dominating density, discussed for instance in [33, Section 14.3.1], can be applied leading to the following random sampling procedure.

It is intuitive that this algorithm should outperform classical rejection from the bounding set $\mathcal{B}$, since more im- 

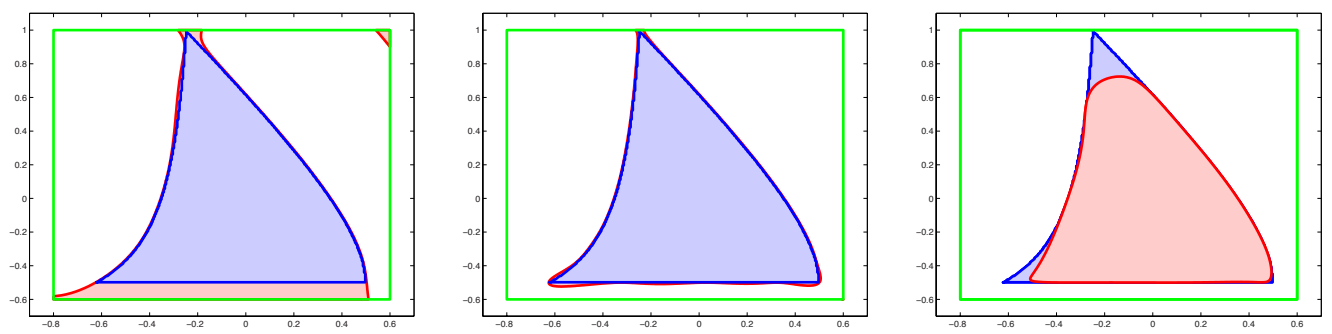

Fig. 2. Degree 6 and degree 20 outer PSS approximation and degree 8 inner PSS approximation (red) of stabilizability region $\mathcal{K}$ (inner surface in light blue). The green box corresponds to the bounding set $\mathcal{B}$.

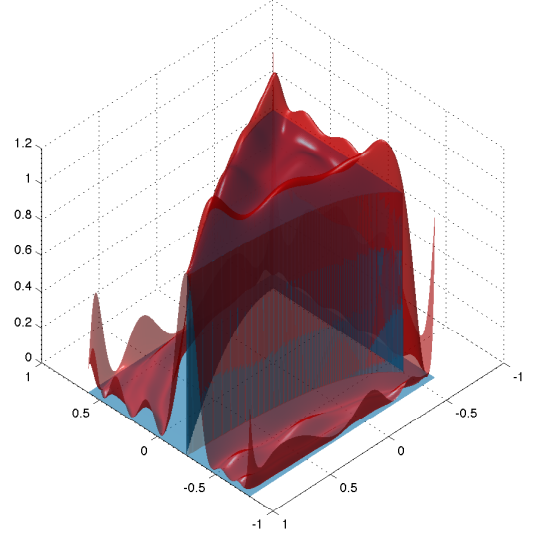

Fig. 3. Degree 20 polynomial approximation (upper surface in red) of the indicator function (lower surface in blue) of the nonconvex planar stabilizability region $\mathcal{K}$.

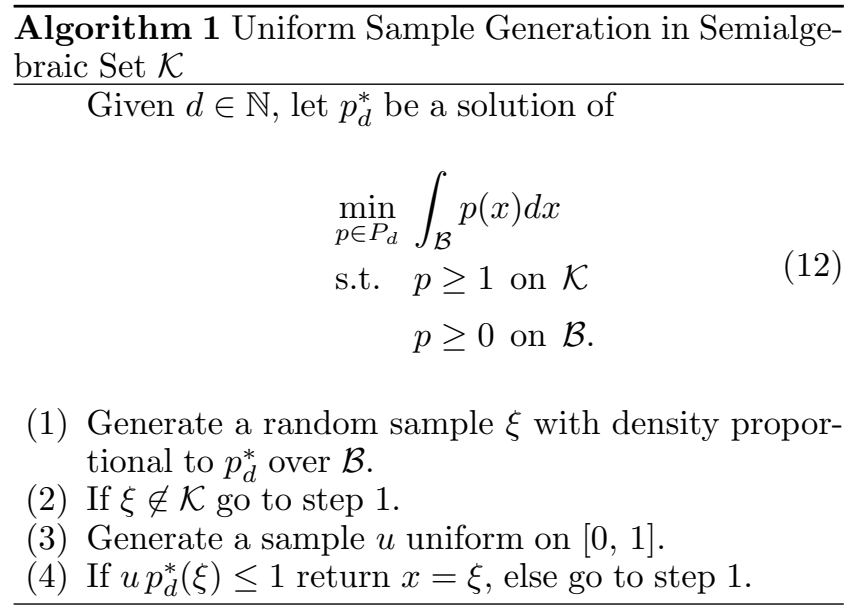

portance is given to the samples inside $\mathcal{K}$ through the function $p_{d}^{*}$. To formally analyze the performance of Algorithm 1, we define the acceptance rate (see e.g. [13]) as the reciprocal of the expected number of samples that have to be drawn from $p_{d}^{*}$ in order to find one "good" sample, that is a sample uniformly distributed in $\mathcal{K}$. Then, the following result, which is the main theoretical result of this section, provides the acceptance rate of the proposed algorithm.

Theorem 4 Algorithm 1 returns a sample uniformly distributed in $\mathcal{K}$. Moreover, the acceptance rate of the algorithm is given by

$$
\gamma_{d}=\frac{\operatorname{vol} K}{w_{d}^{*}},
$$

where $w_{d}^{*} \doteq \int_{\mathcal{B}} p_{d}^{*}(x) d x$ is the optimal solution of problem (7). Then, the acceptance rate tends to one when increasing the degree of the polynomial approximation, i.e. $\lim _{d \rightarrow \infty} \gamma_{d}=1$.

Proof: To prove the statement, we first note that polynomial $p_{d}^{*}$ defines a density

$$
f \doteq \frac{p_{d}^{*}}{w_{d}^{*}}
$$

over $\mathcal{B}$. Moreover, by construction, we have $p_{d}^{*} \geq \mathbb{I}_{\mathcal{K}}$ on $\mathcal{B}$, and hence

$$
\begin{aligned}
\frac{p_{d}^{*}}{w_{d}^{*} \operatorname{vol} \mathcal{K}} & \geq \frac{\mathbb{I}_{\mathcal{K}}}{w_{d}^{*} \operatorname{vol} \mathcal{K}} \\
\frac{f}{\operatorname{vol} \mathcal{K}} & \geq \frac{\mathbb{U}_{\mathcal{K}} f}{w_{d}^{*}} \geq \gamma_{d} \mathbb{U}_{\mathcal{K}}
\end{aligned}
$$

on $\mathcal{B}$. Then, it can be immediately seen that Algorithm 1 is a restatement of the classical Von Neumann rejection algorithm, see e.g. [33, Algorithm 14.2], whose acceptance rate is given by the value of $\gamma_{d}$ such that (14) holds, see for instance [13].

It follows that the efficiency of the random sample generation increases as $d$ increases, and becomes optimal as $d$ goes to infinity. Therefore, a trade-off exists between the complexity of computing a good approximation ( $d$ large) on the one hand, and having to wait a long time to get a "good" sample $(1 / \gamma$ large $)$, on the other hand. Note, 

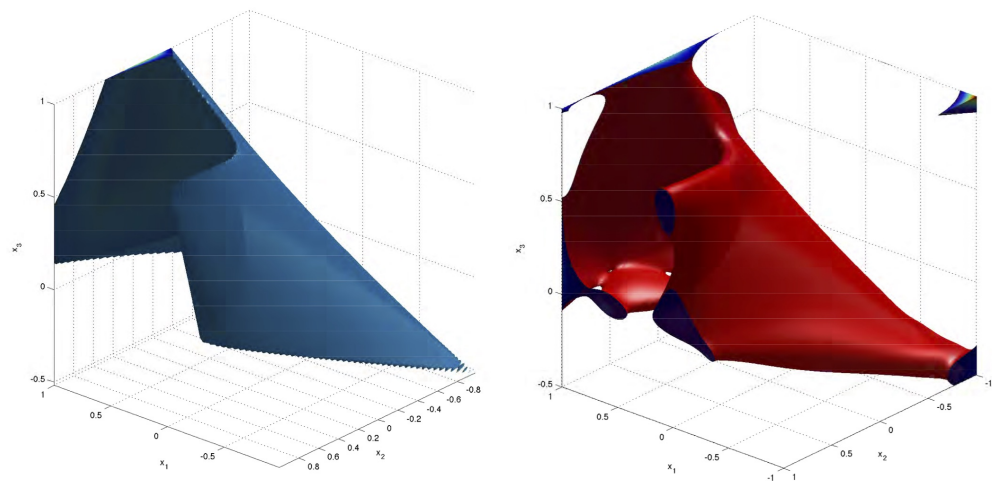

Fig. 4. Left: set of stabilizing PID gains. Right: its degree 14 optimal outer PSS approximation.

however, that the first step can be computed off-line for a given set $\mathcal{K}$, and then the corresponding polynomial $p_{d}^{*}$ can be used for efficient on-line sample generation. Finally, we highlight that, in order to apply Algorithm 1 in an efficient way (step 2), a computationally efficient scheme for generating random samples according to a polynomial density is required. This is discussed next.

\subsection{Sample generation from a polynomial density}

To generate a random sample according to the multivariate polynomial density $f$ defined in (13), one can use the so-called conditional density method described in [13]. This is a recursive method in which the individual entries of the multivariate samples are generated according to their conditional probability density. We now elaborate on this. We should note that the approach developed in this paper only provides the density up to a multiplying constant. However, to simplify the exposition to follow, we proceed as if the polynomial given is indeed a probability density function.

Assume that the bounding set is a hyperrectangle $\mathcal{B}=$ $[a, b]$ of the form (4) and that we have a polynomial density $p$. We start by computing the marginal density

$$
p_{1}: x_{1} \mapsto \int_{a_{2}}^{b_{2}} \cdots \int_{a_{n}}^{b_{n}} p\left(x_{1}, x_{2}, \ldots, x_{n}\right) d x_{2} \cdots d x_{n}
$$

and, for each $i=2, \ldots, n$ and given $\bar{x}_{1}, \ldots \bar{x}_{i-1}$, compute conditional marginal densities

$$
\begin{array}{r}
p_{i}: x_{i} \mapsto \int_{a_{i+1}}^{b_{i+1}} \cdots \int_{a_{n}}^{b_{n}} p\left(\bar{x}_{1}, \ldots, \bar{x}_{i-1}, x_{i}, x_{i+1}, \ldots, x_{n}\right) \\
d x_{i+1} \cdots d x_{n}
\end{array}
$$

and respective (polynomial) cumulative distributions $F_{i}$ satisfying $\frac{d F_{i}}{d x_{i}}=p_{i}$. The sampling procedure then starts by computing a sample $\bar{x}_{1}$ according to $F_{1}$ and, iteratively, computing samples $\bar{x}_{i}$ given $\bar{x}_{1}, \ldots \bar{x}_{i-1}$ according to the distribution $F_{i}$. The exact description of this procedure is described in Algorithm 2. One should note that, given the density $p$, a closed form is available for all marginal and conditional densities. In other words, none of the integrations mentioned above needs to be computed numerically.

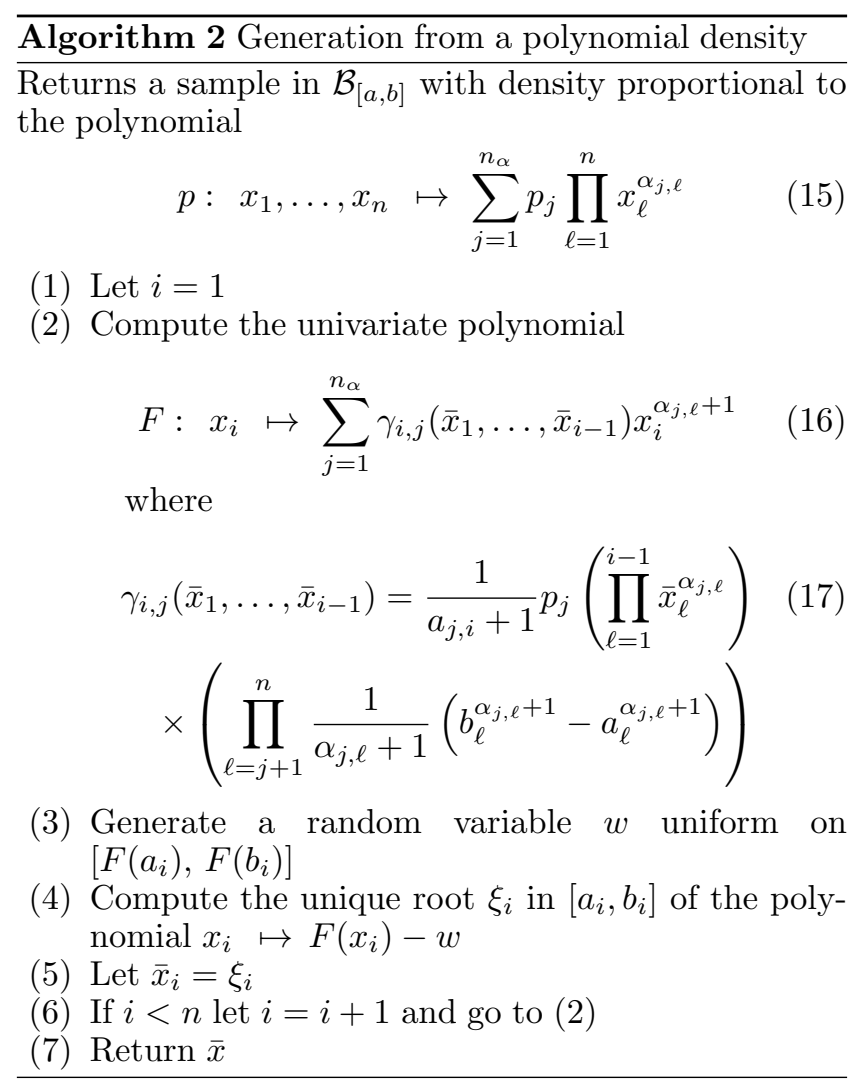

\subsection{Numerical example: sampling in a nonconvex semi- algebraic set}

To demonstrate the behavior of Algorithms 1 and 2, we revisit Example 5.1, and generate uniform samples in the semialgebraic set $\mathcal{K}$ defined in (11). As already shown in Figure 2, the indicator function $\mathbb{I}_{\mathcal{K}}$ is well approximated from above by the optimal PSS $p_{d, d}^{*}$ for $d=20$. The re- 
sults of Algorithm 1 are reported in Figure 5. The red points represent the points which have been discarded. To this regard, it is important to notice that also some point falling inside $\mathcal{K}$ has been rejected. This is fundamental to guarantee uniformity of the discarded points.

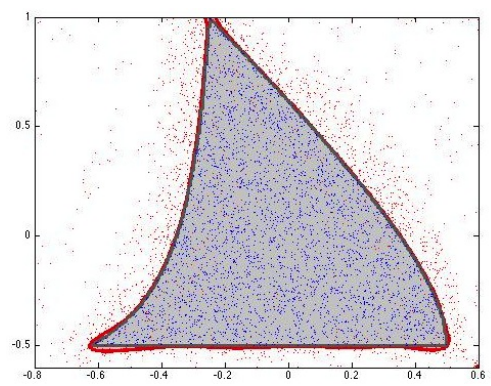

Fig. 5. Uniform random samples generated according to Algorithms 1 and 2 . The light blue area is the set $\mathcal{K}$ defined in (11), the pink area is the PSS. The red dots are the discarded samples. The remaining samples (blue) are uniformly distributed inside $\mathcal{K}$.

\section{Concluding Remarks}

In this paper we have introduced the concept of polynomial superlevel sets (PSS) as a tool to construct "simple" approximations of complex semialgebraic sets. Algorithms are provided for computing these approximations. Moreover, it is shown how this concept can be used to solve two important problems: i) reconstruction/approximation of sets from samples and ii) generation of uniform samples in basic semialgebraic sets. Examples of the application of these ideas to problems in control engineering are also described. Note that the methods provided in this paper can be used to obtain probabilistic approximations of difficult sets, in the spirit of what is discussed in [12]. Also, we note that similar techniques can also be used to approximate transcendental (i.e. non-semi-algebraic) sets arising in systems control, e.g. regions of attraction, maximum positively invariant sets, and controllability regions. Finally, we stress that the focus of the paper is on low degree approximations (say $d<40$ ). The results presented in the paper, proving convergence for high degree, are important in that they testify for the theoretical soundness of the chosen approximation. To this regard, the evaluation of the convergence rate represents an important issue, which we plan to address in our subsequent work.

\section{A Proof of Theorem 1}

As in [20, Section 3.2], let $x \mapsto d(x, \mathcal{K})$ be the Euclidean distance to set $\mathcal{K}$ and with $\epsilon_{k}>0$ let $\mathcal{K}_{\epsilon}:=\{x \in \mathcal{B}$ : $\left.d(x, \mathcal{K})<\epsilon_{k}\right\}$ be an open bounded outer approximation of $\mathcal{K}$, so that $\mathcal{B} \backslash \mathcal{K}_{k}$ is closed with $\lim _{k \rightarrow \infty} \epsilon_{k}=0$. By Urysohn's Lemma [29, Section 12.1] there is a sequence of continuous functions $\left(f_{k}\right)_{k \in \mathbb{N}}$ with $f_{k}: \mathcal{B} \rightarrow[0,1]$ such that $f_{k}=0$ on $\mathcal{B} \backslash \mathcal{K}_{k}$ and $f_{k}=1$ on $\mathcal{K}$. In particular, notice that vol $\mathcal{K} \leq \operatorname{vol} \mathcal{U}\left(f_{k}\right) \leq \operatorname{vol} \mathcal{K}+\operatorname{vol} \mathcal{K}_{k} \backslash \mathcal{K}$ and since $\lim _{k \rightarrow \infty} \operatorname{vol} \mathcal{K}_{k} \backslash \mathcal{K}=0$ it holds $\lim _{k \rightarrow \infty} \operatorname{vol} \mathcal{U}\left(f_{k}\right)=$ $\operatorname{vol} \mathcal{K}$.

By the Stone-Weierstrass Theorem [29, Section 12.3] we can approximate $f_{k}$ uniformly on $\mathcal{B}$ by a sequence of polynomials $\left(p_{k, d}^{\prime}\right)_{d \in \mathbb{N}}$ with $p_{k, d}^{\prime} \in P_{d}$, i.e. $\sup _{x \in \mathcal{B}}\left|f_{k}(x)-p_{k, d}^{\prime}(x)\right|<\epsilon_{d}^{\prime}$ with $\lim _{d \rightarrow \infty} \epsilon_{d}^{\prime}=0$. Defining $p_{k, d}:=p_{k, d}^{\prime}+2 \epsilon_{d}^{\prime}$, the sequence of polynomials $\left(p_{k, d}\right)_{d \in \mathbb{N}}$ converges uniformly to $f_{k}$ from above, i.e. $p_{k, d} \geq f_{k}$ on $\mathcal{B}$ and $\lim _{d \rightarrow \infty} \sup _{x \in \mathcal{B}}\left|f_{k}(x)-p_{k, d}(x)\right|=0$. This implies that $v_{k, d}^{*}:=\operatorname{vol} \mathcal{U}\left(p_{k, d}\right) \geq \operatorname{vol} \mathcal{U}\left(f_{k}\right)$ and $\lim _{d \rightarrow \infty} v_{k, d}^{*}=\operatorname{vol} \mathcal{U}\left(f_{k}\right)$. Recalling $\lim _{k \rightarrow \infty} \operatorname{vol} \mathcal{U}\left(f_{k}\right)=$ vol $\mathcal{K}$, it follows that $\lim _{k, d \rightarrow \infty} v_{k, d}^{*}=\operatorname{vol} \mathcal{K}$ which proves, up to extracting a subsequence indexed by $d$, the existence of a minimizing sequence of polynomials for optimization problem (3). Finally, the inequality $v_{d}^{*} \geq v_{d+1}^{*}$ follows from the inclusion $P_{d} \subset P_{d+1}$.

\section{B Proof of Theorem 2}

Let us first extend optimization problem (7) to continuous functions:

$$
w^{*} \doteq \inf _{f} \int_{\mathcal{B}} f(x) d x \text {, s.t. } f \in \mathcal{C}_{+}(\mathcal{B}), f-1 \in \mathcal{C}_{+}(\mathcal{K})
$$

where $\mathcal{C}_{+}(\mathcal{B})$ denotes the convex cone of non-negative continuous functions on $\mathcal{B}$. Observe that since $f$ is nonnegative on $\mathcal{B}$, the objective function $\|f\|_{1}=\int_{\mathcal{B}} f(x) d x$ is linear. Problem (B.1) is an infinite-dimensional linear programming (LP) problem in cones of non-negative continuous functions. It has a dual LP, in infinitedimensional dual cones of measures:

$$
\begin{aligned}
v^{*} \doteq \sup _{\mu, \hat{\mu}} & \int \mu(d x) \\
\text { s.t. } & \mu(d x)+\hat{\mu}(d x)=\mathbb{I}_{\mathcal{B}}(x) d x \\
& \hat{\mu} \in \mathcal{C}_{+}^{\prime}(\mathcal{B}), \mu \in \mathcal{C}_{+}^{\prime}(\mathcal{K})
\end{aligned}
$$

where $\mathcal{C}_{+}^{\prime}(\mathcal{B})$ is the cone of non-negative continuous linear functionals on $\mathcal{C}_{+}(\mathcal{B})$, identified with the cone of Borel regular non-negative measures on $\mathcal{B}$, according to a Riesz Representation Theorem [29, Section 21.5]. In LP (B.2) the right hand side in the equation is the Lebesgue measure on $\mathcal{B}$. Since the mass of non-negative measures $\mu$ and $\hat{\mu}$ is bounded, it follows from Alaoglu's Theorem on weak-star compactness [29, Section 15.1] that the supremum is attained in dual LP (B.2) and that there is no duality gap between the primal and dual LP, i.e. $v^{*}=w^{*}$, see also e.g. [3, Theorem IV.7.2].

Moreover, as in the proof of [20, Theorem 3.1], it holds $v^{*}=\operatorname{vol} \mathcal{K}$. To see this, notice first that the constraint $\mu+\hat{\mu}=\mathbb{I}_{\mathcal{B}}$ jointly with $\mu \in \mathcal{C}_{+}^{\prime}(\mathcal{K})$ imply that $\mu \leq \mathbb{I}_{\mathcal{K}}$ and hence $\int \mu \leq \int \mathbb{I}_{\mathcal{K}}=$ vol $\mathcal{K}$ for every $\mu$ feasible in LP (B.2). In particular, this is true for an optimal $\mu^{*}$ 
attaining the supremum, showing $\int \mu^{*}=v^{*} \leq \operatorname{vol} \mathcal{K}$. Conversely, the choice $\mu=\mathbb{I}_{\mathcal{K}}$ is trivially feasible for LP (B.2) and hence suboptimal, showing $v^{*} \geq \int \mu=\operatorname{vol} \mathcal{K}$. From this proof it also follows that the only optimal solution to $\operatorname{LP}\left(\right.$ B.2) is the pair $\left(\mu^{*}, \hat{\mu}^{*}\right)=\left(\mathbb{I}_{\mathcal{K}}, \mathbb{I}_{\mathcal{B} \backslash \mathcal{K}}\right)$. Now let us prove the statements of the Theorem: i) Attainment of the infimum in problem (7) follows from continuity (actually linearity) of the objective function $\|p\|_{1}=\int_{\mathcal{B}} p(x) d x=0$ which is a norm (i.e. $\|p\|_{1}=0$ implies $p=0$ for $p \in P_{d}$ ) and compactness of the set $\left\{p \in P_{d}: p \in \mathcal{C}(\mathcal{B}),\|p\|_{1} \leq r\right\}$ for any fixed $r>0$; ii) $w_{d}^{*} \geq v_{d}^{*}$ follows readily from $(6)$; iii) $w_{d}^{*} \geq w_{d+1}^{*}$ follows readily from $P_{d} \subset P_{d+1}$. Finally, $\lim _{d \rightarrow \infty} w_{d}^{*}=\operatorname{vol} \mathcal{K}$ is a consequence of $v^{*}=w^{*}=\operatorname{vol} \mathcal{K}$ (proven above) and the Stone-Weierstrass Theorem [29, Section 12.3] allowing to approximate uniformly on $\mathcal{B}$ by polynomials any continuous function in a minimizing sequence for LP (B.1), i. e. $\lim _{d \rightarrow \infty} w_{d}^{*}=w^{*}$.

\section{References}

[1] T. Alamo, J.M. Bravo, and E.F. Camacho. Guaranteed state estimation by zonotopes. Automatica, 41(6):1035-1043, 2005.

[2] R.B. Ash and C.A. Doléans-Dade. Probability and measure theory, 2nd edition. Academic Press, San Diego, CA, 2000.

[3] A. Barvinok. A course in convexity. American Mathematical Society, Providence, USA, 2002.

[4] A. Ben-Tal and A. Nemirovski. Lectures on modern convex optimization. SIAM, Philadelphia, PA, 2001.

[5] S.P. Bhattacharyya, Datta A, and L.H. Keel. Linear Control Theory: Structure, Robustness, and Optimization. SpringerVerlag, Boca Raton, 2009.

[6] V. Cerone, D. Piga, and D. Regruto. Polytopic outer approximations of semialgebraic sets. In Proc. of the IEEE Conference on Decision and Control, pages 7793-7798, 2012.

[7] G. Chesi, A. Garulli, A. Tesi, and A. Vicino. Solving quadratic distance problems: An LMI-based approach. IEEE Transactions on Automatic Control, 48:200-212, 2003.

[8] F. Dabbene, P. Gay, and B.T. Polyak. Recursive algorithms for inner ellipsoidal approximation of convex polytopes. Automatica, 39(10):1773-1781, 2003.

[9] F. Dabbene and D. Henrion. Set approximation via minimum-volume polynomial sublevel sets. In Proc. of the European Control Conference, 2013.

[10] F. Dabbene, D. Henrion, and C. Lagoa. Uniform sample generation in semialgebraic sets. In Proc. of the IEEE Conference on Decision and Control, 2014.

[11] F. Dabbene, D. Henrion, C. Lagoa, and P. Shcherbakov. Randomized approximations of the image set of nonlinear discrete-time systems with applications to filtering. In Proc. of the IFAC ROCOND Symposium, 2015.

[12] F. Dabbene, C. Lagoa, and P.S. Shcherbakov. On the complexity of randomized approximations of nonconvex sets. In Proc. of the IEEE Multiconference on Systems and Control, 2010.

[13] L.P. Devroye. Non-Uniform Random Variate Generation. Springer-Verlag, New York, 1986.

[14] C. Durieu, B.T. Polyak, and E. Walter. Trace versus determinant in ellipsoidal outer-bounding with application to state estimation. In Proc. IFAC World Congress, San Francisco, CA, 1996.
[15] C. Durieu, E. Walter, and B.T. Polyak. Multi-input multioutput ellipsoidal state bounding. Journal of Optimization Theory and Applications, 111(2):273-303, 2001.

[16] A. Garulli, A. Tesi, and A. Vicino. Robustness in Identification and Control. Springer-Verlag New York, Inc., Secaucus, NJ, USA, 1999.

[17] L. El Ghaoui and G. Calafiore. Robust filtering for discretetime systems with bounded noise and parametric uncertainty. IEEE Transactions on Automatic Control, 46(7):1084-1089, July 2001.

[18] L.J. Guibas, An Nguyen, and Li Zhang. Zonotopes as bounding volumes. In SODA '03: Proc. of the Fourteenth Annual ACM-SIAM Symposium on Discrete Algorithms, pages 803-812, 2003.

[19] D. Henrion and J.B. Lasserre. Inner approximations for polynomial matrix inequalities and robust stability regions. IEEE Transactions on Automatic Control, 57(6):1456-1467, 2012.

[20] D. Henrion, J.B. Lasserre, and C. Savorgnan. Approximate volume and integration for basic semialgebraic sets. SIAM Review, 51(4):722-743, 2009.

[21] D. Henrion and C. Louembet. Convex inner approximations of nonconvex semialgebraic sets applied to fixed-order controller design. International Journal of Control, 85(8):1083-1092, 2012.

[22] V. Kuntsevich and M. Lychak. Synthesis of optimal and adaptative control systems: The game approach. Naukova Dumka, (in Russian), 1985.

[23] J.B. Lasserre. Global optimization with polynomials and the problem of moments. SIAM J. Optim., 11(3):796-817, 2001.

[24] J. Lofberg. Yalmip : A toolbox for modeling and optimization in MATLAB. In Proc. of the CACSD Conference, 2004.

[25] A. Magnani, S. Lall, and S.P. Boyd. Tractable fitting with convex polynomials via sum-of-squares. In Proc. IEEE Conf. on Decision and Control and Europ. Control Conf., Sevilla, Spain, 2005.

[26] A. Morozov and S. Shakirov. New and old results in resultant theory. Teoreticheskaya i Matematicheskaya Fizika, 163(2):222-257, 2010.

[27] P.A. Parrilo. Exploiting structure in sum of squares programs. In Proc. of the IEEE Conference on Decision and Control, 2003.

[28] M. Putinar. Positive polynomials on compact semi-algebraic sets. Indiana University Mathematics Journal, 42(3):969984, 1993.

[29] H. L. Royden and P. M. Fitzpatrick. Real Analysis. 4th Edition. Prentice Hall, 2010.

[30] F.C. Schweppe. Uncertain Dynamical Systems. PrenticeHall, Englewood Cliffs, 1973.

[31] J.S. Shamma and K.-Y. Tu. Approximate set-valued observers for nonlinear systems. IEEE Transactions on Automatic Control, 5:648-658, 1997.

[32] J.F. Sturm. Using SeDuMi 1.02, a MATLAB toolbox for optimization over symmetric cones. Optimization Methods and Software, 11-12:625-653, 1999.

[33] R. Tempo, G.C. Calafiore, and F. Dabbene. Randomized Algorithms for Analysis and Control of Uncertain Systems: With Applications. Springer, 2nd edition, 2013.

[34] J. M. Wojciechowski and J. Vlach. Ellipsoidal method for design centering and yield estimation. Transactions on Computer-Aided Design of Integrated Circuits and Systems, 12:1570-1579, 1993 